\title{
Um modelo de localização e transbordo multiproduto para avaliação do impacto de regimes aduaneiros
}

\author{
Irineu de Brito Jr. ${ }^{1}$, Patrícia Belfiore ${ }^{2}$ e Hugo Tsugunobu Yoshida Yoshizaki ${ }^{3}$
}

Resumo: Este artigo analisa o impacto dos regimes aduaneiros na logística de aquisição de materiais produtivos em unidades fabris instaladas no Brasil e no exterior, para uma empresa fabricante de aviões comerciais e militares. Primeiramente, são avaliadas as alterações dos custos logísticos proporcionados pelos regimes aduaneiros RECOF e "Drawback". Em seguida, um modelo de localização e transbordo multiproduto é aplicado para determinação dos locais de consolidação e para otimização dos custos logísticos; verificam-se quais produtos seguem diretamente dos fornecedores, localizados na Europa e Estados Unidos, para a unidade consumidora na China, e quais sofrem etapa de transbordo no Brasil, onde existem restrições de capacidade na armazenagem. A aplicação é realizada em empresa aeronáutica com atividades internacionais em Harbin (norte da China) e os resultados demonstram que os regimes aduaneiros, principalmente em função do valor do produto, influenciam os custos logísticos e a consolidação dos materiais.

DOI:10.4237/transportes.v20i3.624.

Palavras-chave: localização de instalações, modelo de transbordo, regimes aduaneiros, tributos, indústria aeronáutica.

\begin{abstract}
This research studies the impact of customs system regime in a logistics network design for a manufacturer of commercial and military aircrafts with plants installed in Brazil and abroad and with international activities in Harbin (China). Firstly, we evaluate the changes in the logistics costs provided by two tax regimes: RECOF and Drawback. Then, a location and transshipment model with multi-product is applied to optimize the logistics costs and to determine where the goods should be consolidated. The use of facility locations to transport different products from various sources to various destinations can take advantage of economies of scale in transportation costs. Instead of making direct shipments, each source can ship in bulk to consolidation terminals. The application is held in aerospace company with international activities in Harbin (northern China) and the results show that the tax regime, mainly due to the value of the product, affects significantly logistics costs and goods consolidation. Thus, it is crucial to understand the referred tax regimes, to avoid severe logistics network system sub-optimization.
\end{abstract}

Keywords: facility location; transshipment model; taxes, customs systems, aircraft industry.

\section{INTRODUÇÃO}

Este artigo tem como objetivo analisar o impacto dos regimes aduaneiros na logística de materiais produtivos em $\mathrm{u}$ nidades fabris instaladas no Brasil e no exterior, para uma empresa fabricante de aviões comerciais e militares. A descentralização das operações industriais da empresa torna complexo o seu abastecimento, principalmente com o início da operação de novas unidades no exterior, as quais recebem materiais dos Estados Unidos, Europa e Brasil.

O transporte aéreo é uma indústria cíclica altamente sensível a flutuações na economia, que requer o uso intensivo de capital e mão-de-obra para aquisições, operação e manutenção de aeronaves e, por possuir ciclos logísticos muito longos, requer ajustes rápidos nos processos produtivos, assim como no apoio aos clientes, incluindo a alta competitividade entre as companhias aéreas. Essa necessidade de resposta rápida conflita com os ciclos de entrega de novas aeronaves que, geralmente, são longos. Tal característica exige uma antecipação de demanda e, consequentemente,

\footnotetext{
${ }^{1}$ Irineu de Brito Jr, Fatec São José dos Campos e Departamento de Engenharia de Produção, Escola Politécnica, Universidade de São Paulo, São Paulo, SP, Brasil. (e-mail: ibritojr@usp.br).

${ }^{2}$ Patrícia Belfiore, Departamento de Engenharia de Gestão, Universidade Federal do ABC, Santo André, SP, Brasil. (e-mail: patricia.favero@ufabc. edu.br).

${ }^{3}$ Hugo Tsugunobu Yoshida Yoshizaki, Departamento de Engenharia de Produção e Programa de Sistemas Logísticos, Escola Politécnica, Universidade de São Paulo, São Paulo, SP, Brasil. (e-mail: hugo@usp.br).
}

Manuscrito recebido em 6/8/2012 e aprovado para publicação em 2/10/2012. Este artigo é parte de TRANSPORTES v. 20, n. 3, 2012. ISSN: 2237-1346 (online). DOI:10.4237/transportes.v20i3.624. de compras por parte das companhias aéreas. Desta forma, o mercado do transporte aéreo exige dos fabricantes de aeronaves uma cadeia de suprimentos flexível para absorver as oscilações deste mercado e uma constante troca de informações com os parceiros e fornecedores, a fim de minimizar os impactos negativos e/ou maximizar a rentabilidade sobre as vendas. A flexibilidade das linhas produtivas para assimilar adiamentos e acelerações de pedidos, bem como a postergação da configuração de características diferenciadas e específicas das aeronaves, instaladas conforme solicitação do cliente, são também exigências para a cadeia de suprimentos. Estes aspectos, juntamente com o projeto do produto, podem ser tratados como diferenciais competitivos das montadoras de aeronaves.

A indústria aeronáutica no Brasil possui desafios, principalmente na logística de aquisição, transportando materiais dos fornecedores às unidades produtivas. Seus fornecedores e clientes localizam-se nos Estados Unidos, Europa e Extremo Oriente. Este quadro passa a ser ainda mais complexo com a instalação de unidades fabris na China e a possibilidade de iniciar a fabricação nos Estados Unidos.

O problema estudado pode ser modelado através de programação linear inteira mista, onde o objetivo é minimizar os custos logísticos totais, identificando quais produtos seguirão diretamente dos fornecedores para a unidade consumidora e quais produtos sofrerão uma etapa de transbordo em um centro de consolidação de materiais. O problema consiste em encontrar a localização ótima dos polos consolidadores, a partir de um conjunto de locais candidatos, de forma que a demanda dos clientes seja atendida, o custo total de transporte e armazenagem seja minimizado. Este 
problema é classificado como um problema de localização e transbordo ou produção-distribuição multiproduto (twostage production-distribution system design problem with multi-commodity - TSPDSDP with multi-commodity).

\section{REVISÃO DA LITERATURA}

Melo et al (2009) destacam que decisões de localização de instalações possuem papel crítico na concepção estratégica das Cadeias de Suprimentos e caracterizam o problema de localização de instalações como um conjunto de clientes espacialmente distribuídos e um conjunto de instalações para atender demandas destes clientes. Daskin (2008) apresenta uma taxonomia para o problema de localização subdivindo o problema em modelos analíticos, contínuos, de rede e discretos. Vasconcelos et al (2010) utilizam a teoria de grafos como ferramenta fundamental na metodologia de localização, em especial a aplicação de fluxos em rede.

\subsection{O problema de localização e transbordo e suas extensões}

De acordo com Matisziw (2005), o problema de transbordo (Transshipment Problem) é uma extensão do problema clássico de transporte onde, ao invés de transportar os produtos diretamente de várias fontes para vários destinos, trabalha-se com pontos intermediários de transbordo (instalações), que podem ligar esses caminhos a fim de reduzir os custos logísticos. Para Keskin e Üster (2007a), o problema de transbordo considera três elos na cadeia de abastecimento, onde o processo de transporte ocorre em dois estágios: o transporte de fornecedores aos pontos de transbordo e transporte dos pontos de transbordo aos pontos de demanda. O objetivo do problema de transbordo é determinar o fluxo de mercadorias provenientes de um conjunto de origens para um conjunto de destinos, através de um conjunto de nós intermediários com o objetivo de minimizar o custo total de transporte no sistema.

Outro problema logístico importante, amplamente estudado na literatura, é o de localização de instalações (Facility Location Problem - FLP). O termo instalações inclui fábricas, depósitos, aeroportos, portos marítimos, terminais etc. O objetivo do problema de localização é determinar o número de instalações, selecionar a(s) localização(ões) ótima(s) dentre possíveis locais candidatos e atribuir os clientes às mesmas, visando satisfazer a demanda com menor custo. Se as instalações têm capacidade limitada, o problema é chamado problema de localização de instalações com capacidade limitada (Capacitated Facility Location Problem - CFLP). Caso contrário, tem-se um problema de localização de instalações com capacidade irrestrita (Uncapacitated Facility Location Problem - UFLP).

O TSCFLP (Two-Stage Capacitated Facility Location Problem) é uma extensão do CFLP, considerando dois estágios (três elos na cadeia de surprimentos). O TSCFLP é também uma extensão do problema de transbordo, onde os locais dos pontos de transbordo devem ser escolhidos (problema de localização e transbordo simultaneamente). O problema tem como objetivo localizar $p$ pontos de transbordo e determinar a transferência de produtos dos fornecedores aos clientes por esses pontos. Na primeira etapa, o produto é transportado das instalações dos fornecedores para os pontos de transbordo, cujas posições devem ser determinadas. Na segunda etapa, as demandas dos clientes devem ser satisfeitas por estas instalações. O objetivo do problema é minimizar o custo variável de entrega e os custos operacionais fixos (Elhedhli; Goffin, 2005). O TSCFLP também pode ser classificado na literatura como um problema de produção-distribuição de dois estágios (Two Stage Production Distribution System Design Problem TSPDSDP) que, de acordo com Keskin e Üster (2007b), envolve a determinação da melhor configuração, com relação à localização e o tamanho das plantas e centros de distribuição, a oferta de produtos e as decisões de transporte. Quando houver mais de três estágios da cadeia de abastecimento, o problema é chamado de problema de localização de instalação com capacidade limitada multiestágio (MSCFLP) ou problema de produção-distribuição com capacidade limitada multi-estágio (MSPDSDP).

Keskin e Üster (2007b) apresentaram diferentes classificações para o CFLP ou PDSDP (Production Distribution System Design Problem). Alguns trabalhos são baseados em dois estágios, enquanto outros consideram modelos com três ou mais estágios. Outro aspecto importante a ser considerado é o nível dos locais de instalações. Além disso, o número de instalações pode ou não ser limitado. Ou ainda, em alguns trabalhos, a capacidade das instalações e/ou a capacidade da fábrica são limitados, mas em outros não. Outro ponto importante é a fonte de distribuição, que pode ser única ou múltipla. $\mathrm{Na}$ fonte única, cada cliente é atribuído a uma única facilidade, enquanto em fontes múltiplas cada cliente pode ser atribuído a mais de uma instalação. Alguns modelos consideram custos fixos das instalações e fábricas, outros não. Finalmente, alguns problemas consideram apenas um produto, enquanto outros diversos produtos. Dasci e Verter (2001) também consideram outras variáveis em sua taxonomia, como função objetivo (custo, lucro ou múltipla), tipo de demanda (determinística ou estocástica) e o número de períodos a ser considerado (único ou múltiplos períodos).

\subsection{Métodos de solução para o problema de localização e transbordo}

Embora exista uma grande quantidade de estudos na literatura sobre problemas de localização e distribuição, a maioria baseia-se em um único estágio; pesquisas com dois ou múltiplos estágios são limitadas. Os principais trabalhos relacionados ao problema estudado serão descritos a seguir. Primeiramente, serão descritos os trabalhos baseados em multiprodutos.

Um dos primeiros trabalhos na área é o de Geoffrion e Graves (1974). Propuseram a decomposição de Benders para resolver o problema de produção-distribuição de dois estágios multiprodutos. Hindi e Basta (1994) e Hindi et al. (2006) recomendam um algoritmo branch-and-bound para o mesmo problema, enquanto Pirkul e Jayaraman (1996, 1998) propõem uma heurística baseada em relaxação lagrangeana para resolver o TSPDSDP multiproduto. Já os autores Keskin e Üster (2007a) recomendam a metaheurística "busca dispersa" para resolver um TSPDSDP com número fixo de instalações intermédiárias que devem ser localizadas entre as plantas e os clientes. Os mesmos autores apresentaram, em 2007b, uma abordagem híbrida da metaheurística "busca dispersa", e resolveram o TSPDSDP multiproduto.

Lee (1991, 1996) e Mazzola e Neebe (1999) também estudaram o TSPDSDP multiproduto. Lee $(1991,1996)$ pro- 
pôs um algoritmo baseado em decomposição de Benders para resolver o modelo. Mazzola e Neebe utilizaram um algoritmo branch-and-bound e uma heurística baseada em relaxação lagrangeana para o problema. O problema de produção-distribuição multiproduto com três estágios pode ser encontrado em Jayaraman e Pirkul (2001) e Syarif et al. (2002). Tcha e Lee (1984) consideram mais de três estágios.

Dentre os trabalhos sobre PDSDP baseados em apenas um produto, destacam-se: Marín e Pelegrin (1997, 1999), Tragantalerngsak et al. (1997, 2000), Klose (1999, 2000), Elhedhli e Goffin (2005). Marín e Pelegrin (1997, 1999) propõem um algoritmo branch-and-bound e relaxação lagrangeana para resolver o TSPDSDP. Tragantalerngsak et al. $(1997,2000)$ propuseram uma heurística lagrangeana e algoritmo branch-and-bound, respectivamente, para resolver o problema de localização capacitado de dois estágios e uma única fonte. Klose (1999) utilizou uma heurística baseada em programação linear para o problema de localização de instalações capacitado de dois estágios. Em 2000, Klose propôs um algoritmo lagrangeano baseado na relaxação das restrições de capacidade da fábrica e do depósito para o problema de localização capacitado de dois estágios com uma única fonte, resultando em um problema de localização de instalações com capacidade irrestrita (UFLP). O resultado do subproblema lagrangeano foi resolvido por métodos branch-and-bound. Elhedhli e Goffin (2005) utilizaram relaxação lagrangeana, métodos de ponto interior e algoritmo branch and bound para resolver o TSPDSDP. A maioria dos trabalhos sobre PDSDP utiliza programação linear inteira mista para modelar o problema. Dasci e Verter (2001) apresentaram um modelo contínuo alternativo baseado na utilização de funções contínuas para representar distribuições espaciais de custo e demanda do cliente.

Finalmente, considerando os parâmetros propostos por Keskin e Üster (2007b) e Dasci e Verter (2001), o problema estudado neste trabalho tem as seguintes características: rede em dois estágios; localização de centros de distribuição; número limitado de instalações; capacidade dos fornecedores e centros de distribuição limitados; cada cliente pode ser atribuído a mais de uma instalação (multisourcing); o modelo considera os custos fixos para cada local de consolidação; multiproduto; demanda determinística; e período único. Além disso, diferentemente da maioria dos trabalhos encontrados na literatura, considera-se a alternativa de fazer transferências diretas dos fornecedores aos clientes, sem pontos de transbordo (modelos de fase única). O problema estudado neste trabalho é classificado como um problema de produção-distribuição de dois estágios multiproduto (multi-product TSPDSDP).

\subsection{Regimes Aduaneiros}

\subsubsection{Regime aduaneiro especial de Drawback}

O benefício de Drawback é um incentivo à exportação e compreende a suspensão, isenção ou restituição de tributos incidentes na importação de mercadoria utilizada na industrialização de produto exportado ou a exportar. Esses impostos compreendem o Imposto de Importação (II); o Imposto sobre Produtos Industrializados (IPI); o Imposto sobre Operações relativas à Circulação de Mercadorias e sobre a Prestação de Serviços de Transporte Interestadual e Intermunicipal e de Comunicação (ICMS), conforme defi- nido pelos Estados e Distrito Federal; e o Adicional ao Frete para Renovação da Marinha Mercante (AFRMM). As importações amparadas no regime não estão sujeitas ao exame de similaridade nem à obrigatoriedade de transporte em navio de bandeira brasileira.

Entende-se por industrialização qualquer operação que modifique a natureza, o funcionamento, o acabamento, a apresentação ou a finalidade do produto, ou o aperfeiçoe para consumo, tais como: a transformação, o beneficiamento que modifica o acabamento, a montagem que resulta em um novo produto, a renovação ou recondicionamento para utilização, e o acondicionamento ou recondicionamento que altere a apresentação do produto.

A legislação prevê três modalidades de aplicação de Drawback:

1. Suspensão: nesta modalidade o benefício é aplicado sob a forma de suspensão do pagamento de tributos devidos sobre importação de mercadoria a ser exportada. Compreende:

- Imposto de Importação;

- Imposto sobre Produtos Industrializados;

- Imposto sobre Operações relativas à Circulação de Mercadorias e sobre Prestação de Serviços de Transporte Interestadual e Intermunicipal e de Comunicação; e

- Adicional de Frete para Renovação da Marinha Mercante.

2. Isenção: nesta modalidade, a empresa terá direito a importar a mesma quantidade e qualidade de insumos empregados na produção de um produto comprovadamente já exportado. Trata-se de uma reposição de estoque. A isenção compreende:

- Imposto de Importação;

- Imposto sobre Produtos Industrializados; e

- Adicional ao Frete para Renovação da Marinha Mercante.

3. Restituição: esta modalidade de refere-se à devolução total ou parcial de tributos que incidiram sobre a importação anterior de mercadoria que venha a ser exportada após industrialização. Compreende:

- Imposto de Importação; e

- Imposto sobre Produtos Industrializados.

Ressalta-se que a restituição dos tributos é concedida sob a forma de crédito fiscal à importação, a ser utilizado em qualquer importação posterior.

$\mathrm{O}$ embarque da mercadoria para o exterior deve ser efetuado no prazo máximo de um ano, podendo ser prorrogado respeitando-se o limite de dois anos.

\subsubsection{Regime RECOF}

O regime RECOF (Regime Aduaneiro de Entreposto Industrial sob Controle Informatizado) foi instituído pela Secretaria da Receita Federal em Dezembro de 1997 e visa proporcionar ganhos de custo e tempo no processo aduaneiro, possibilitando a suspensão/isenção de impostos, juntamente com uma grande agilidade no processo logístico. A suspensão dos impostos incidentes na importação criou flexibilidade e vantagem competitiva nunca vista em nenhum outro regime aduaneiro especial, em função da vantagem financeira para o fluxo de caixa das empresas.

Pode-se mencionar que as empresas capazes de desfrutar do regime são as que: 
- Realizam processos de industrialização, montagem ou transformação;

- Tenham idoneidade fiscal;

- Exportam ou participam de cadeia produtiva exportadora;

- Assumam compromisso de limite mínimo de exportações, de acordo com a modalidade de seus negócios;

- Possuam software de controle que atenda as exigências da SRF - RECOF Sys; e

- Tenham patrimônio líquido superior a R\$ 2 milhões.

O pilar básico do regime RECOF é a confiança que a Receita Federal coloca na empresa habilitada, permitindo que a empresa realize processos de liberação sem a inspeção da Receita Federal nas áreas de desembaraço, tendo todos os processos sempre parametrizados no canal verde, bem como a possibilidade de importar as mercadorias com suspensão dos impostos. Esses impostos somente serão pagos no momento da venda do produto final, mesmo que seja vendido no mercado local. Estima-se que todos esses benefícios podem levar a empresa a ter um ganho de $2 \%$ a $6 \%$ do total importado, conforme a operação.

Segundo Portugal (2003), além de prover consultas em tempo real para a Receita Federal, o software é também responsável por todo o controle operacional sobre o regime RECOF, fornecendo funcionalidades que realizarão o controle do prazo de permanência no regime, controle da estrutura de produção, controle dos processos de perdas no processo produtivo, verificando a necessidade ou não do pagamento dos impostos, controle dos processos administrativos de destruição e apuração ou não do pagamento de todos os impostos suspensos devido à importação sob o regime.

\subsubsection{Recof Aeronáutico}

O RECOF para a Indústria Aeronáutica é concedido pela Secretaria da Receita Federal do Ministério da Fazenda. Dentre as operações de industrialização, destacam-se: 1) modalidades de montagem de aeronaves, equipamentos e instrumentos de uso aeronáutico; 2) transformação, beneficiamento e montagem de partes, peças e componentes utilizados na produção, além da montagem de instrumentos de uso aeronáutico; 3 ) mercadorias que poderão estar inclusas neste regime, e serão aplicadas no desenvolvimento de produtos da indústria aeronáutica; 4) produtos da indústria aeronáutica que serão submetidos a operações de renovação, recondicionamento, reparo e manutenção; e 5) produtos da indústria aeronáutica que serão submetidos a testes de funcionamento. A exportação da mercadoria sob regime de RECOF deve ser efetuada no prazo máximo de um ano, prorrogável por mais um.

As vantagens do regime Recof em comparação ao regime Drawback são:

- Permite destinar até $20 \%$ do valor importado anualmente, para exportação ou mercado interno sem que ocorra a transformação (industrialização);

- Não há limitação de valor para importação;

- Suspensão de impostos para todos os insumos elegíveis, não somente para os destinados à exportação;

- Definição de quantidade destinada à exportação $a$ posteriori;

- Elimina burocracia (ato concessório, licença técnica, comprovações, etc.);
- Facilita o trânsito de produtos com os fornecedores, através da eliminação da necessidade de Drawback intermediário;

- Admite tolerância de perdas no processo produtivo (scrap isento);

- Não exige segregação física dos produtos importados sob o regime;

- Processo de importação mais rápido (canal verde), que proporciona a redução do ciclo aduaneiro de desembaraço de cinco dias para seis horas e, consequentemente, a redução das despesas de armazenagem em zona aduaneira; e

- Controle por somente um órgão do Governo Federal (Receita Federal), sem o envolvimento do Decex.

Dentre as desvantagens do Recof em relação ao Drawback, destacam-se:

- Os custos de implantação do regime, que exigem altos investimentos em sistemas de informação e na adequação das operações; e

- Tornar disponível à Receita Federal as informações online sobre todos os materiais estocados ou em processo.

\section{DEFINIÇÃO E MODELAGEM DO PROBLEMA}

\subsection{Definição do problema}

Este estudo se aplica aos materiais enviados para abastecimento da linha de produção localizada em Harbin (norte da China), os quais possuem restrições de lote mínimo de aquisição resultante de uma quantidade mínima para compra ou restrição de embalagem.

Os materiais são adquiridos de fornecedores situados nos Estados Unidos e Europa e, para abastecimento da linha de produção em Harbin, podem ser enviados diretamente para a unidade ou sofrer uma etapa de consolidação no Brasil (São José dos Campos), nas Costas Leste ou Oeste dos Estados Unidos (Miami e Los Angeles) ou ainda na França (Paris).

Devido à característica de lote mínimo de aquisição, deve-se ponderar o trade off entre o custo financeiro de manutenção desses estoques na unidade de consumo mais o frete de abastecimento direto, versus o abastecimento desta unidade, na quantidade exata de sua necessidade a partir de outro local, onde ocorrem custos adicionais de transporte, manuseio e custos fixos para instalação e operação de um centro de transbordo (polo de consolidação). Os polos de consolidação (depósitos) possuem restrições de capacidade máxima e mínima. $\mathrm{O}$ custo de manutenção dos estoques ocorre devido ao aumento dos níveis de estoques para abastecer a unidade China. Na unidade São José dos Campos já ocorre a compra do lote mínimo para atendimento da atual demanda. Caso a consolidação do material não ocorra nesta unidade, outro lote mínimo deve ser adquirido, ocasionando aumento nos estoques na unidade de destino ou em outro polo consolidador. A Figura 1 mostra o comportamento dos estoques em cada unidade, permitindo concluir que o custo financeiro corresponde à diferença do custo médio do capital imobilizado em cada uma das situações.

O problema é analisado utilizando-se cenários, comparados a partir de regimes tributários e modais de transporte. Sob o aspecto tributário, a análise é a alteração dos custos 

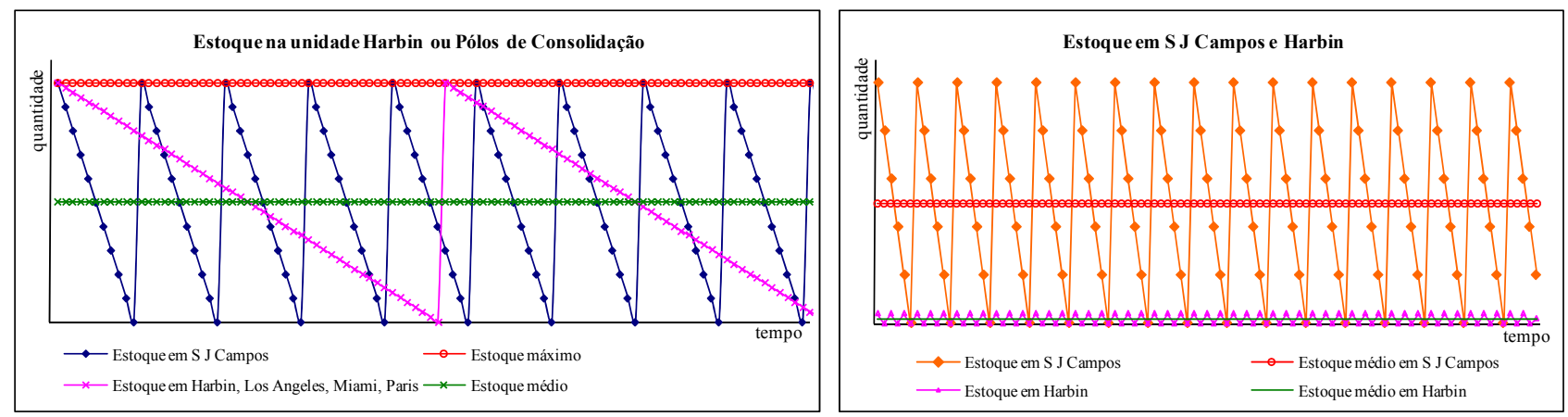

Figura 1. Comportamento dos estoques

logísticos a partir da implementação de um novo regime fiscal, denominado RECOF Aeronáutico, que está substituindo o atual benefício de Drawback. Quanto ao modal de transporte, consideraram-se os modais aéreo e marítimo para transporte dos materiais do fabricante ao polo consolidador ou à unidade de montagem final.

As informações sobre o problema modelado estão resumidas a seguir:

- Horizonte de planejamento: refere-se à produção de 100 aeronaves em Harbin;

- Origem: os materiais são produzidos nos Estados Unidos e Europa;

- Locais candidatos para a instalação de polo consolidador (depósito): São José dos Campos, Los Angeles, Miami e Paris; e

- Destino: todos os produtos são destinados à linha de produção situada em Harbin.

O objetivo é encontrar o número, o tamanho e as localizações de depósitos em uma rede logística que minimizarão os custos fixos e variáveis que movimentarão todos os produtos através da rede selecionada, sujeito às seguintes condições:

1. A demanda de todos os produtos deve ser satisfeita;

2. Um processamento mínimo de um depósito deve ser alcançado antes que ele possa ser aberto (restrição contratual com operador logístico no exterior);

3. O processamento de cada depósito não pode exceder sua capacidade;

4. A capacidade de fornecimento não pode ser excedida para cada produto.
Neste problema, cada cliente pode ser atribuído a mais de uma instalação (multiproduto). Além disso, diferentemente da maioria dos trabalhos encontrados na literatura, considera-se a alternativa de fazer transferências diretas dos fornecedores aos clientes, sem pontos de transbordo. $\mathrm{Na}$ Figura 2, a seguir, são mostrados os fluxos e locais de envios dos materiais.

\subsection{Modelagem matemática}

O problema estudado é modelado através de programação linear inteira mista e tem como base os trabalhos de Hindi e Basta (1994) e Keskin e Üster (2007). Têm-se os seguintes índices:

- Fornecedores $i \in I$

- Os fornecedores encontram-se nos Estados Unidos e Europa.

- Polos consolidadores $j \in J$

- São locais candidatos à abertura ou não de polo consolidador (depósito). Estes locais são:

- São José dos Campos (SJK - Brasil) - operação pela empresa, restrição de capacidade máxima;

- Los Angeles (LAX - costa oeste dos EUA) operação por terceiros, restrição de capacidade mínima;

- Miami (MIA - costa leste dos EUA) - operação por terceiros, restrição de capacidade mínima;

- Paris (CDG - Europa) - operação por tercei-

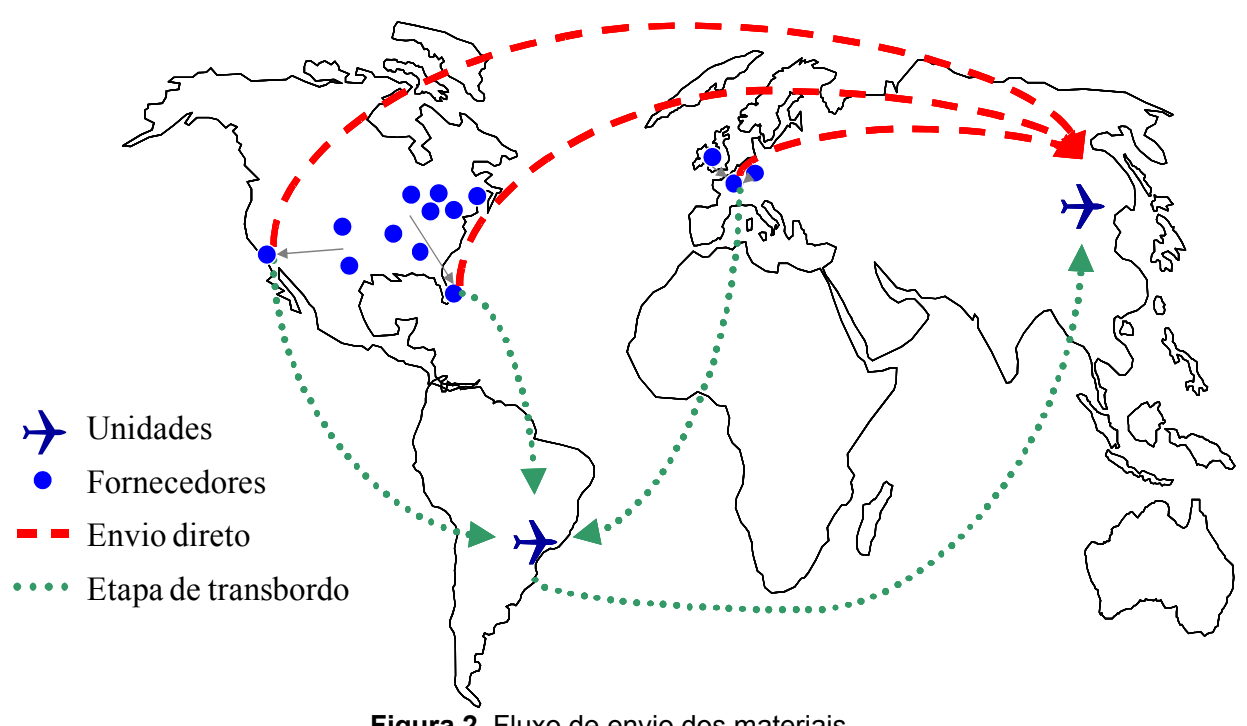

Figura 2. Fluxo de envio dos materiais 
ros, restrição de capacidade mínima;

- Harbin (HRB - China) - operação pela empresa. A abordagem da unidade China como polo consolidador foi adotada com o objetivo de estabelecer limites para a quantidade enviada sem a etapa de transbordo. $O$ custo da transferência deste polo para a unidade de destino é zero.

- Produtos $p \in P$

- São os produtos que serão ou não consolidados. São 256 itens aeronáuticos que possuem lote mínimo de fornecimento que excede o lote econômico de compra. O modelo de estoque é reativo, pois o pedido de novos materiais ocorre no período imediatamente após a quantidade estocada atingir o ponto de pedido. Os fornecedores são únicos por uma questão comercial, embora o modelo seja capaz de considerar mais de um fornecedor.

- O local de destino dos produtos é a fábrica de Harbin (China).

Os principais parâmetros do modelo de programação linear inteira mista estão listados a seguir.

- $Q_{j}$ : capacidade máxima do polo consolidador $j$ em $\mathrm{kg} /$ ciclo.

- $q_{j}$ : quantidade mínima para abertura do polo consolidador $j$ em kg/ciclo.

- $f_{j}$ : custo fixo para abertura do depósito $j$, em US\$/ciclo.

- $D_{p}$ : demanda do produto $p$ na fábrica em $\mathrm{kg} / \mathrm{ciclo}$.

- $S_{i p}$ : capacidade do fornecedor $i$ produzir o produto $p$ em kg/ciclo.

- $g_{p j}$ : custo de transbordo por unidade do produto $p$ no polo consolidador $j$, em US $\$ / \mathrm{kg}$.

- $\quad c_{p i j}$ : custo unitário de transporte de $p$ do fornecedor $i$ para polo consolidador $j$, em US $\$ / \mathrm{kg}$.

- $\quad c_{p j}$ : custo unitário de transporte de $p$ do polo consolidador $j$ para a fábrica, em US\$ $/ \mathrm{kg}$.

- $\quad c_{p i}$ : custo unitário de transporte de $p$ do fornecedor $i$ para a fábrica, em US $\$ / \mathrm{kg}$.

As variáveis de decisão do modelo são:

- $x_{p i j}$ : quantidade $(\mathrm{kg})$ transferida do produto $p$ do fornecedor $i$ para o polo consolidador $j$.

- $y_{p j}$ : quantidade $(\mathrm{kg})$ transferida do produto $p$ do polo consolidador $j$ para a fábrica.

- $\mathrm{z}_{p i}$ : quantidade $(\mathrm{kg})$ transferida do produto $p$ do fornecedor $i$ para a fábrica.

- $\mathrm{z}_{j}$ : variável binária que assume valor 1 caso o polo $\mathrm{j}$ opere e 0 em caso contrário.

O problema pode ser formulado da seguinte forma:

$$
\begin{aligned}
& \min \sum_{p} \sum_{i} \sum_{j} c_{p i j} x_{p i j}+\sum_{p} \sum_{j} c_{p j} y_{p j}+ \\
& +\sum_{p} \sum_{i} c_{p i} z_{p i}+\sum_{p} \sum_{j} g_{p j} y_{p j}+\sum_{j} f_{j} z_{j}
\end{aligned}
$$

Sujeito a:

$$
\sum_{j} y_{p j}+\sum_{i} z_{p i}=D_{p} \quad \forall p
$$

$$
\begin{array}{cc}
\sum_{p} \sum_{i} x_{p i j} \leq Q_{j} z_{j} & \forall j \\
\sum_{p} \sum_{i} x_{p i j} \geq q_{j} z_{j} & \forall j \\
\sum_{j} x_{p i j}+z_{p i} \leq S_{i p} & \forall i, p \\
\sum_{i} x_{p i j}=y_{p j} & \forall p, j \\
x_{p i j}, y_{p j}, z_{p i} \geq 0 & \forall p, i, j \\
z_{j} \in\{0,1\} & \forall z
\end{array}
$$

$\mathrm{Na}$ função objetivo, o primeiro termo representa os custos de transporte dos fornecedores até terminais de consolidação, o segundo refere-se aos custos de transporte dos terminais de consolidação para o cliente final (fábrica de Harbin), o terceiro representa os custos de transporte dos fornecedores diretamente ao cliente final, e os dois últimos os custos fixos operacionais e de localização de terminais de consolidação, respectivamente.

A restrição (1) garante que a demanda pelo produto $p$ no cliente é atendida. A restrição (2) é relacionada com a capacidade máxima de cada terminal de consolidação. A quantidade mínima para a abertura de um terminal de consolidação é garantida pela restrição (3). A restrição (4) representa a capacidade de oferta do fornecedor i para o produto p. (5) é a restrição de conservação de fluxo. Finalmente, as restrições (6) e (7) são de não-negatividade e as restrições de integralidade.

\subsubsection{Custos logísticos considerados na operação}

Para a apuração, os custos logísticos foram divididos em três grandes grupos, sendo:

- Custos de Transbordo:

- Tributos: Imposto de Importação (II), Imposto sobre Produtos Industrializados (IPI), Imposto sobre Operações relativas à Circulação de Mercadorias e sobre Prestação de Serviços de Transporte Interestadual e Intermunicipal e de Comunicação (ICMS), Adicional de Frete para Renovação da Marinha Mercante (AFRMM);

- Custos de desembaraço aduaneiro;

- Custos de movimentação do material em zona aduaneira;

- Custos de recebimento, inspeção, armazenagem e movimentação de material; e

- Custos financeiros de manutenção dos estoques.

- Custos de Transporte:

- Frete aéreo ou marítimo;

- Seguro;

- Custos de transporte (zona primária para depósito na importação e trajeto de retorno na exportação do material); e

- Custo do estoque em trânsito ("pipeline”). 


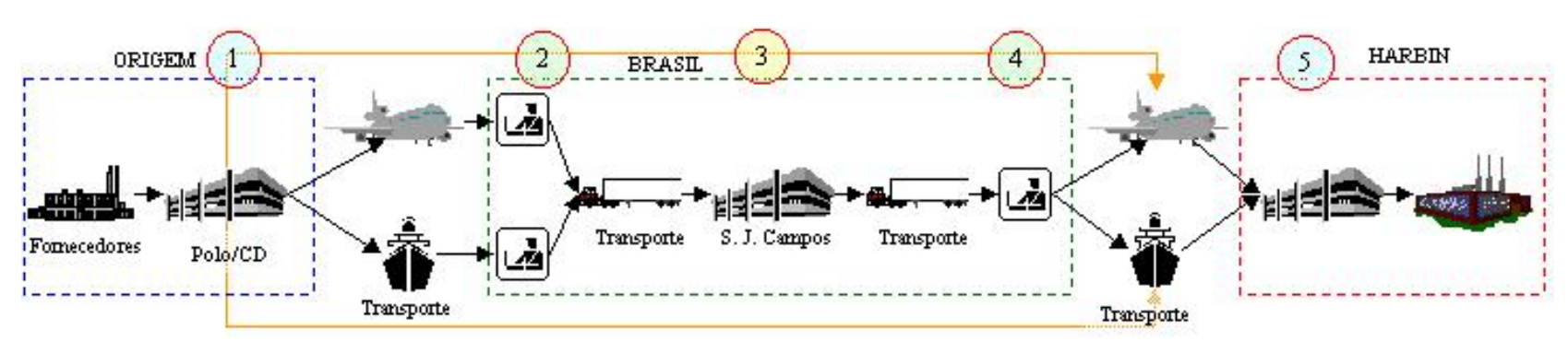

Legenda:

1 - Origem dos materiais (Los Angeles, Miami, Paris);

2 - Zona Primária no Brasil (porto ou aeroporto) na importação;

3 - Unidade São José dos Campos;

4 - Zona Primária no Brasil (porto ou aeroporto) na exportação;

5 - Unidade Harbin (depósito).

Figura 3. Fluxo de materiais e locais de ocorrência de custos

Tabela 1. Custos

\begin{tabular}{|c|c|c|c|c|c|c|}
\hline Tipo & Parâme tro & $\begin{array}{l}\text { Ocorrência } \\
\text { (Fig 3) }\end{array}$ & Custo & Peso & Valor & Quantidade \\
\hline Transbordo & $\mathrm{gp}, \mathrm{j}$ & 3 & Tributo - Imposto de Importação & & $\mathrm{X}$ & \\
\hline Transbordo & $\mathrm{gp}, \mathrm{j}$ & 3 & Tributo - IPI & & $\mathrm{X}$ & \\
\hline Transbordo & $\mathrm{gp}, \mathrm{j}$ & 3 & Tributo - ICMS & & $\mathrm{X}$ & \\
\hline Transbordo & $g p, j$ & 2 & Tributos - AFRMM & $\mathrm{X}$ & $\mathrm{X}$ & \\
\hline Transbordo & $\mathrm{gp}, \mathrm{j}$ & 2 & Desembaraço aduaneiro & & & $\mathrm{X}$ \\
\hline Transbordo & $\mathrm{gp}, \mathrm{j}$ & 4 & Infraero exportação & $\mathrm{X}$ & & \\
\hline Transbordo & gp,j & 2 & Infraero importação & & $\mathrm{X}$ & \\
\hline Transbordo & $\mathrm{gp}, \mathrm{j}$ & 2 & Capatazia & $\mathrm{X}$ & $\mathrm{X}$ & \\
\hline Transbordo & $\mathrm{gp}, \mathrm{j}$ & 3 & $\begin{array}{l}\text { Recebimento, Armazenagem e Ex- } \\
\text { pedição }\end{array}$ & & & $\mathrm{X}$ \\
\hline Transporte & $\begin{array}{l}c p, \mathrm{i}, \mathrm{j} \\
\mathrm{cp}, \mathrm{j}, \mathrm{k} \\
\mathrm{cp}, \mathrm{i}, \mathrm{k}\end{array}$ & $\begin{array}{l}1-2 \\
4-5 \\
1-5 \\
\end{array}$ & Frete aéreo & $\mathrm{X}$ & & \\
\hline Transporte & $\begin{array}{l}\text { cp,i,j } \\
\text { cp,j,k } \\
\text { cp,i,k }\end{array}$ & $\begin{array}{l}1-2 \\
1-5 \\
\end{array}$ & Frete marítimo & $\mathrm{X}$ & & \\
\hline Transporte & $\mathrm{cp}, \mathrm{j}, \mathrm{k}$ & $\begin{array}{l}2-3 \\
3-4 \\
\end{array}$ & Seguro nacional & & $\mathrm{X}$ & \\
\hline Transporte & $\begin{array}{l}c p, \mathrm{i}, \mathrm{j} \\
\mathrm{cp}, \mathrm{j}, \mathrm{k} \\
\mathrm{cp}, \mathrm{i}, \mathrm{k}\end{array}$ & $\begin{array}{l}1-2 \\
4-5 \\
1-5 \\
\end{array}$ & Seguro internacional & & $\mathrm{X}$ & \\
\hline Transporte & $\begin{array}{l}\text { cp,i,j } \\
\text { cp,j,k } \\
\text { cp,i,k }\end{array}$ & $\begin{array}{l}1-2 \\
4-5 \\
1-5\end{array}$ & Estoque em trânsito & & $\mathrm{X}$ & \\
\hline Transbordo & $\mathrm{cp}, \mathrm{j}$ & $\begin{array}{l}1 \\
5\end{array}$ & Custo financeiro do estoque & & $\mathrm{X}$ & \\
\hline
\end{tabular}

- Custo fixo para instalação (ou abertura) de depósito.

Obs.: Acordos comerciais no segmento aeronáutico entre Brasil e China ou entre os países de origem dos materiais e a China, que possam criar vantagens comerciais que alterem a apuração dos custos logísticos, não foram estabelecidos até a presente data. A Figura 3 apresenta o fluxo de mercadorias na cadeia de suprimentos estudada e os locais de ocorrência dos custos.

Direcionadores e locais de ocorrência dos custos:

Os custos referentes ao produto (transbordo e transporte) são levantados em função do peso do produto, valor do produto ou simplesmente em função do item de forma unitária.

A Tabela 1 mostra, para cada custo considerado, este direcionador para apuração.

\subsection{Solução e análise dos resultados}

Para resolver o problema de programação linear inteira mista proposto aplicou-se o software GAMS ("General Algebric Modelling System”), utilizando diversos solvers que são executados pelo programa. Dentre estes solvers possíveis de serem utilizados destacam-se o CPLEX (da ILOG) e o OSL (da IBM). No processo de validação foram testados estes dois solvers, utilizando todos os materiais (256) em duas situações, com erro o absoluto igual a 0 (zero) e também com um erro relativo igual a 0,000001 . Os resultados mostraram desvios quando estabelecido o erro absoluto

Tabela 2. Cenários

\begin{tabular}{lll}
\hline & Descrição do cenário & $\begin{array}{l}\text { Regime } \\
\text { Tributário }\end{array}$ \\
\hline Cenário 1 & Envio direto dos materiais a Harbin \\
\hline Cenário 2 & & Recof \\
Cenário 3 & Modal Aéreo & Drawback \\
\hline Cenário 4 & & Recof \\
Cenário 5 & Modais Marítimo e Aéreo & Drawback \\
\hline
\end{tabular}




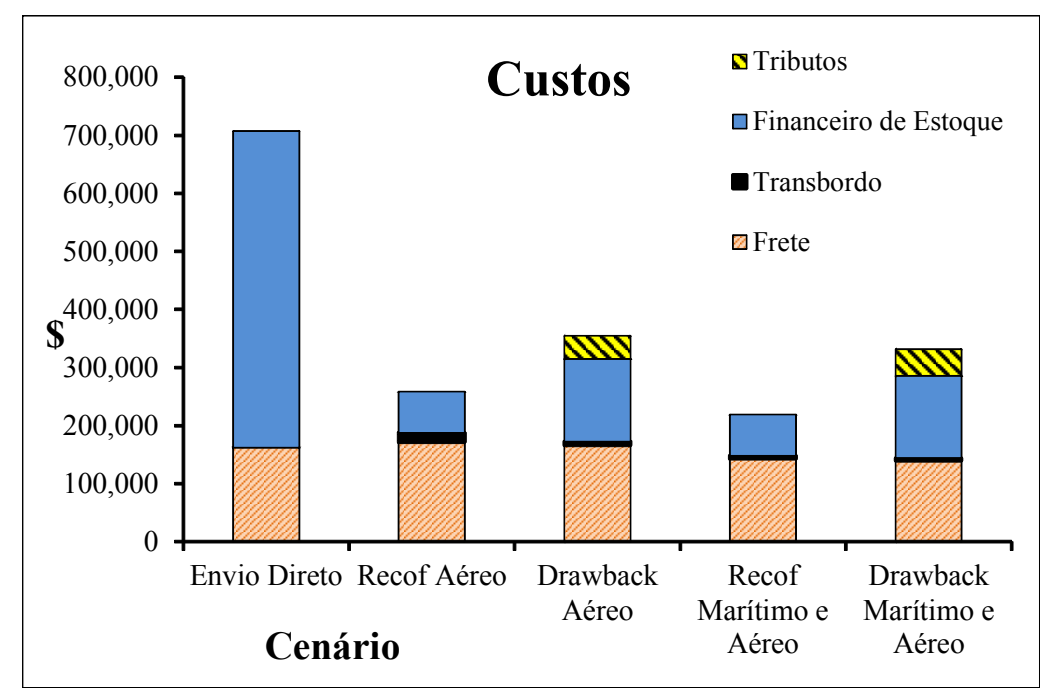

Figura 4. Resumo da composição dos custos em cada um dos cenários

para o "solver" OSL. Decidiu-se não utilizar esta situação para este solver e utilizar somente o erro relativo.

Os cenários apresentados na Tabela 2 foram considerados para estudo em função de representarem as possibilidades de ocorrência dos modais de transporte e do regime tributário.

- Cenário 1: abastecimento direto: este cenário era a proposição logística inicial, considerando todos os materiais seguindo diretamente para a unidade Harbin, neste cenário não são aplicados os tributos tipicamente brasileiros.

- Cenário 2 e 3: modal aéreo em todas etapas de transporte.

- Cenário 4 e 5: o transporte do fornecedor até o polo consolidador é realizado através do modal marítimo e do polo até a unidade consumidora pelo modal aéreo.

Os resultados mostraram que em todos os cenários houve a utilização do polo consolidador. A Figura 4 apresenta os custos em cada um dos cenários avaliados. Volumes e custos unitários não são mostrados por questão de sigilo de dados. Pode-se observar que os custos financeiros de estoque são os maiores fatores de influência na variação do custo total:

O valor de Custo Financeiro do Estoque no regime
Drawback foi maior que em regime Recof em função dos tributos incidirem sobre o valor do produto. Desta forma, em alguns casos foi viável enviar o produto diretamente a Harbin e arcar com os custos de estoque em vez da incidência de impostos no Brasil.

\subsection{Análise de sensibilidade}

A análise de sensibilidade foi verificada para três parâmetros:

- Variação do fator de peso das embalagens, devido às operações encontrarem-se no início tem-se um baixo número de embarques, o que compromete o histórico para o cálculo desta média;

- Crescimento do valor do frete Brasil-China, atualmente o frete Brasil-China é inferior ao ChinaBrasil. A análise realizada é quanto à consolidação dos itens pode ser alterada e qual o impacto no custo total de um aumento do valor do frete Brasil-China; e

- Alterações no plano de produção, considerando-se duas situações: na primeira considera-se acréscimo e decréscimo nas vendas de 50\%. Na segunda situação será considerado o aumento da cadência de produção durante o período de 100 (cem) meses.

Alterações nos parâmetros: variação do fator de peso das

\section{Variação do Cus to Total}

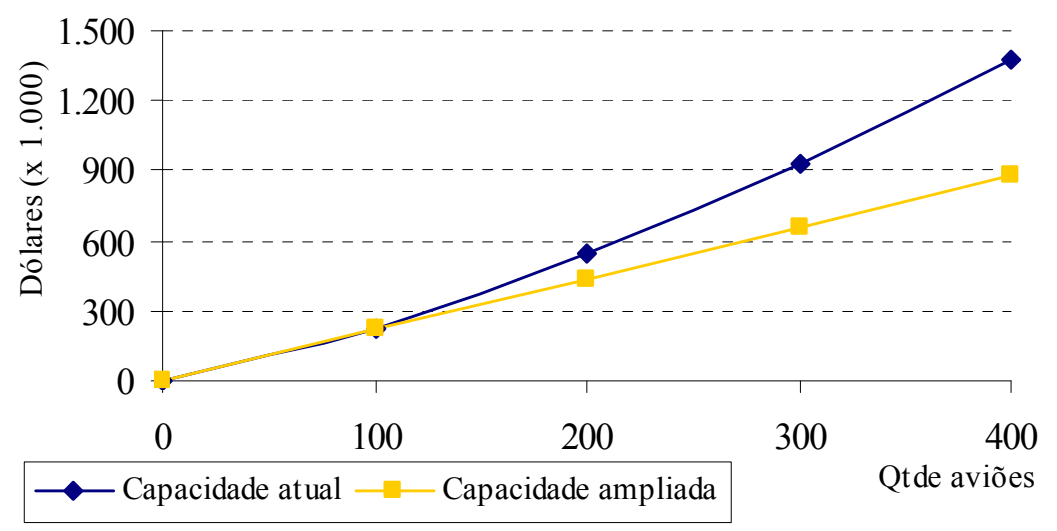

Figura 5. Comparativo de custos: Capacidade Atual x Capacidade Ampliada 
embalagens e crescimento do valor do frete Brasil-China não afetaram significativamente a consolidação dos materiais. O parâmetro alterações nas vendas de aeronaves pode afetar significativamente a consolidação dos materiais. A Figura 5 mostra a variação do custo logístico a partir do aumento das vendas de aeronaves, ampliando a capacidade de transbordo em São José dos Campos ou mantendo-se a capacidade atual, mostrando que a diferença dos valores passa a justificar o aumento da capacidade na unidade São José dos Campos.

\subsection{Implementação do modelo}

A operação da unidade China iniciou-se em 2004 com uma proposta inicial de produção 100 aeronaves até a metade de 2011. Em 2006, a produção atingiu sua cadência nominal. O regime Recof iniciou-se efetivamente em 2008, entretanto a consolidação de materiais foi considerada desde o início das operações segundo o regime Recof. No período de 2006 até 2008, apesar da operação sobre regime Drawback, foi obedecida a consolidação apresentada para o regime Recof, principalmente pelo motivo da não alteração de contratos com fornecedores internacionais. Dos 256 itens 145 seguiram diretamente e 111 realizaram a etapa de transbordo.

\section{CONCLUSÕES}

Objetivando verificar as possíveis alterações na consolidação dos materiais, desenvolveu-se uma análise do impacto de regimes aduaneiros na logística internacional. Esta análise foi desenvolvida a partir da construção de um modelo de programação linear inteira mista que otimizou os custos de transporte, transbordo e financeiro dos materiais que possuem lote mínimo superior ao lote econômico de compra.

Estudos complementares ao modelo também evidenciaram que o tamanho do lote e o custo do produto afetam diretamente a consolidação de materiais. Quanto maior o tamanho do lote, menor o impacto da etapa de transbordo no custo total. Produtos com alto valor agregado e lotes de aquisição maiores realizaram a etapa de transbordo, enquanto produtos de baixo valor e pequenos lotes não realizaram a etapa de transbordo.

Por meio dos resultados, observa-se que a mudança nos regimes aduaneiros pode afetar a logística de consolidação dos materiais, não existindo uma correlação entre os materiais que passam por esta consolidação nas duas situações. Isso ocorre devido aos critérios de tributação que variam conforme a classificação físcal do material. No regime Recof, a quantidade de materiais com etapa de transbordo no Brasil é superior à quantidade no regime Drawback, resultado já esperado devido aos tributos que não incidem no regime.

Estas mudanças nos regimes aduaneiros ocorrem devido ao esforço governamental para facilitar as exportações e também para facilitar a fiscalização realizada pela Receita Federal. Mudanças no regime aduaneiro que possam ocorrer durante um processo em operação podem comprometer todo o processo logístico e levar a profundas alterações na logística para abastecimento ou distribuição.

Além de proporcionar ganhos com a redução dos custos logísticos, o regime aduaneiro do Recof Aeronáutico promove também vantagens na estratégia competitiva dos usu- ários, através da redução dos ciclos logísticos e dos lead times de abastecimento, proporcionando uma maior flexibilidade nas linhas de produção e, consequentemente, uma capacidade maior de atendimento às solicitações dos compradores (clientes).

\section{REFERÊNCIAS BIBLIOGRÁFICAS}

Dasci, A. e V. Verter (2001) A continous model for productiondistribution system design. European Journal of Operational Research, v. 129, n. 2, p. 287-298. DOI: 10.1016/S0377-2217(00) $\underline{00226-5}$

Daskin, M. S. (2008) What You Should Know About Location Modeling, Naval Research Logistics, v. 55, p. 283-294. DOI: 10.1002/ $\underline{\text { nav. } 20284}$

Elhedhli, S. e J. L. Goffin (2005) Efficient Production-Distribution System Design. Management Science, v. 51, n. 7, p. 1151-1164. DOI: $10.1287 / \mathrm{mnsc} .1050 .0392$.

Geoffrion, A. M. e G.. W. Graves (1974) Multicommodity distribution design by Benders decomposition. Management Science, v. 20, n. 5 , p. $822-844$. DOI: $10.1287 / \mathrm{mnsc} .20 .5 .822$.

Hindi, K.S. e T. Basta (1994) Computationally efficient solution of a multiproduct, two-stage distribution-location problem. The Journal of the Operational Research Society, v. 45, n. 11, p. 1316-1323. DOI: $10.1057 /$ jors.1994.204.

Hindi, K.S., T. Basta e K. Piénkosz (2006) Efficient solution of a multicommodity, two-stage distribution problem with constraints on assignment of customers to distribution centers. International Transactions in Operations Research, v. 5, n. 6, p. 519-527. DOI: 10.1016/S0969-6016(98)00039-2.

Jayaraman, H. e H. Pirkul (2001) Planning and coordination of production and distribution facilities for multiple commodities. European Journal of Operational Research, v. 133, p. 394-408. DOI: 10.1016/S0377-2217(00)00033-3.

Keskin B. B. e H. Üster (2007a) A Scatter Search-Based Heuristic to Locate Capacitated Transshipment Points. Computers \& Operations Research, v. 34, n. 10, p. 3112-3125. DOI: 10.1016/j.cor.2005. $\underline{11.020 .}$

Keskin B. B. e H. Üster (2007b) Meta-heuristic approaches with memory and evolution for a multi-product production/distribution system design problem. European Journal of Operational Research, v. 182, n. 2, p. 663-682. DOI: 10.1016/j.ejor.2006.07.034.

Klose, A. (1999) An LP-based heuristic for two-stage capacitated facility location problems. The Journal of the Operational Research Society, v. 50, n. 2, p.157-166. DOI: 10.1057/palgrave.jors. 2600675 .

Klose, A. (2000) A Lagrangean relax-and-cut approach for the two-stage capacitated facility location problem. European Journal of $\mathrm{Op}$ erational Research, v. 126, n. 2, p. 408-421. DOI: 10.1016/S0377-2217(99)00300-8.

Lee, C. Y. (1991) An optimal algorithm for the multiproduct, capacitated facility locatin problem with a choice of facility type. Computers \& Operations Research, v. 18, n. 2, p. 167-182. DOI: 10.1016/ 0305-0548(91)90087-8.

Lee, C. Y. (1996) An algorithm for a two-staged distribution system with various types of distribution centers. INFOR, v. 34 , n. 2, p. 105 117.

Marín, A.e B. Pelegrín (1997) A branch-and-bound algorithm for the transportation problem with location of p-transshipment points. Computers \& Operations Research, v. 24, n. 7, p. 659-678. DOI: 10.1016/S0305-0548(96)00078-0.

Marín, A., Pelegrín, B. (1999) Applying Lagrangian relaxation to the resolution of two-stage location problems. Annals of Operations Research, v. 86, p. 179-198. DOI: 10.1023/A:1018998500803.

Matisziw, T. C. (2005) Modeling transnational surface freight flow and border crossing improvement. Dissertation (Master), Ohio State University.

Mazzola, J. B. e A. W. Neebe (1999) Lagrangian-relaxation-based solution procedures for a multiproduct capacitated facility location problem with choice of facility type. European Journal of Operational Research, v. 115, n. 2, p. 285-299. DOI: 10.1016/S03772217(98)00303-8.

Melo M. T., S. Nickel e F. Saldanha-da-Gama (2009) Facility location and supply chain management - A review. European Journal of $\mathrm{Op}$ erational Research, v. 196, p. 401-412, 2009. DOI: 10.1016/ j.ejor.2008.05.007. 
Pirkul, H. e V. Jayaraman (1998) A multi-commodity, multi-plant, capacitated facility location problem: formulation and efficient heuristic solution. Computers \& Operations Research, v. 25, n. 10, p. 869 878. DOI: 10.1016/S0305-0548(97)00096-8,

Pirkul, H. e N. V. Jayarama (1996) Production, transportation, and distribution planning in a multi-commodity tri-echelon system. Transportation Science, v. 30, n. 4, p. 291-302. DOI: 10.1287/ trsc.30.4.291.

Portugal, U. P. (2003) Recof e os ganhos logísticos. Disponível em: $<$ http://www.tradeworks.com.br/portal imprensa/noticia_15. htm>. (Acesso em 10/12/2003).

Syarif, A., Y. Yun e M. Gen (2002) Study on multi-stage logistic chain network: A spanning tree-based genetic algorithm approach. Computers \& Industrial Engineering, v. 43, p. 299-314. DOI: 10.1016/S0360-8352(02)00076-1.

Tcha, D. e B. Lee (1984) A branch and bound algorithm for the multi-level uncapacitated facility location problem. European Journal of $\mathrm{Op}$ erational Research, v. 18, p. 35-43. DOI: 10.1016/03772217(84)90258-3.

Tragantalerngsak, S., J. Holt e M. Rönnqvist (1997) Lagrangian heuristics for the two-echelon, single-source, capacitated facility location problem. European Journal of Operational Research, v. 102, p. 611-625. DOI: 10.1016/S0377-2217(96)00227-5.

Tragantalerngsak, S., J. Holt e M. Rönnqvist (2000) An exact method for the two-echelon, single-source, capacitated facility location problem. European Journal of Operational Research, v. 123, n. 3, p. 473-489. DOI: 10.1016/S0377-2217(99)00105-8.

Vasconcelos, A. D., C. D. Nassi e L. A. S. Lopes (2010) O problema de localização de terminais concentradores e sua aplicação nas redes de transporte intermodal do país. Transportes, v. 18, n. 2, p. $17-$ 27.

Yoshizaki, H. T. Y. (2002) Projeto de redes de distribuição física considerando a influência do imposto de circulação de mercadorias e serviços. Tese (Livre-Docência). Escola Politécnica, Universidade de São Paulo. São Paulo. 